\title{
HS0702+6043 - A new large amplitude sdB variable at the cool end of the instability region ${ }^{\star}$
}

\author{
S. Dreizler ${ }^{1}$, S. L. Schuh ${ }^{1}$, J. L. Deetjen ${ }^{1}$, H. Edelmann ${ }^{2}$, and U. Heber ${ }^{2}$ \\ 1 Institut für Astronomie und Astrophysik, Universität Tübingen, Sand 1, 72076 Tübingen, Germany \\ e-mail: schuh@, deetjen@astro.uni-tuebingen.de \\ 2 Dr.-Remeis-Sternwarte, Universität Erlangen-Nürnberg, Sternwartstr. 7, 96049 Bamberg, Germany \\ e-mail: heber@, edelmann@sternwarte.uni-erlangen.de
}

Received 15 October 2001 / Accepted 4 February 2002

\begin{abstract}
We report on time series photometry of hot sdB stars from the Hamburg Schmidt survey carried out at the Calar Alto $1.23 \mathrm{~m}$ and $2.2 \mathrm{~m}$ telescopes. Among 14 spectroscopically selected candidates we detected oscillations in the sdB star HS0702+6043 with a period of $363 \mathrm{~s}$ and an amplitude of 29 mmag which is among the largest amplitude observed in sdBV stars. A second period of $382 \mathrm{~s}$ with a significantly smaller amplitude $(3.8 \mathrm{mmag})$ might be present. Our NLTE model atmosphere analysis of the time-averaged optical spectra, obtained at the Calar Alto $3.5 \mathrm{~m}$ telescope, indicates that HS0702+6043 has $T_{\text {eff }}=28400 \mathrm{~K}$ and $\log g=5.35$. This places the star at the cool end of the theoretical sdBV instability strip (EC 14026 stars). Among the other thirteen sdB stars we confirmed the variablity of the previously discovered sdBV HS0815+4243 and identified twelve stars to be stable, for which we give upper limits for light variations.
\end{abstract}

Key words. stars: subdwarfs - stars: oscillations - stars: individual: HS0702+6043 - stars: fundamental parameters - stars: horizontal branch

\section{Introduction}

Subdwarf B stars dominate the population of faint blue stars in our own Galaxy. Moreover, considerable evidence has accumulated that they are sufficiently common to be the most likely source for the "UV upturn phenomenon" observed in elliptical galaxies and galaxy bulges (Yi et al. 1997).

SdB stars form a homogeneous group, populating an extension of the Horizontal Branch in the $\left(T_{\mathrm{eff}}-\log g\right)-$ diagram towards higher temperatures up to $40000 \mathrm{~K}$. Following ideas outlined by Heber (1986), the sdB stars can be identified with models for extreme Horizontal Branch stars (EHB) burning He in their core, yet with a very thin $(<4 \%$ by mass) and therefore inert hydrogen envelope. An EHB star bears great resemblance to a helium main-sequence star of half a solar mass, and its

Send offprint requests to: S. Dreizler, e-mail: dreizler@astro.uni-tuebingen.de

* Based on observations collected at the German-Spanish Astronomical Center, Calar Alto, operated by the Max-PlanckInstitut für Astronomie Heidelberg jointly with the Spanish National Commission for Astronomy. further evolution should proceed similarly, i.e. directly to the white dwarf graveyard (Dorman et al. 1993).

The origin of sdB stars however remains largely a puzzle, but evidence is accumulating that close binary evolution is fundamental to the problem (Maxted et al. 2001; Saffer et al. 2001; Heber et al. 2002). They are being formed during the evolution of low and intermediate mass stars, but must have suffered an unusually high mass loss on the Red Giant Branch in order to end up with their hydrogen rich envelope reduced to below $0.02 M_{\odot}$. The life time on the EHB $\left(\sim 10^{8} \mathrm{yr}\right)$ is long compared to other phases of stellar evolution at such high effective temperatures. This is the reason why, in stellar populations like elliptical galaxies, where the luminous blue main sequence stars are long gone, the sdB contribute significantly to the UV flux (UV upturn).

Recently, Charpinet et al. (1996) predicted pulsations in $\mathrm{sdB}$ stars driven by the $\kappa$-mechanism due to an opacity bump caused by iron. Kilkenny et al. (1997) discovered that several sdB stars do indeed pulsate, and they were named EC 14026 variables after the prototype. Today about two dozen EC 14026 variables, or sdBVs, are known. A recent compilation by O'Donoghue et al. (1999) is 
complemented by newest reports from Billères et al. (2000), Piccioni (2000), Silvotti et al. (2000), Brassard et al. (2001), Østensen et al. (2001a), Østensen et al. (2001b), and Ulla et al. (2001). Charpinet (2001) and Charpinet et al. (2001) provide a review of the theoretical modeling of the driving mechanism as well as a confrontation with the most recent observations. Stellar pulsations enable investigations of stellar interiors because the frequencies of the observed eigenmodes directly constrain the physical properties of the stellar structure. As demonstrated in the case of pulsating white dwarfs, asteroseismology can be used to infer stellar parameters like mass, luminosity, thickness of the envelope or rotation, which are difficult to derive from spectroscopic analysis (e.g. Kawaler \& Bradley 1994). In the case of variable sdB stars these parameters will constrain the evolutionary history and therefore shed more light on the origin of these stars.

The seismology of sdBVs is still in its infancy. Before being able to make full use of the potential of the asteroseismology, more basic questions like the extension of the instability strip or the nature of the eigenmodes (radial or non-radial, $p$-mode or $g$-mode pulsations) have to be investigated, and suitable candidates for detailed asteroseismology have to be identified.

A search for pulsating sdBs therefore is a necessary step to enable the investigation of the structure of sdB stars. Several such projects are in progress at the moment (O’Donoghue et al. 1999; Silvotti et al. 2000; Billères et al. 2000; Østensen et al. 2001a,b). In this paper we report on our own observing program.

Without pre-selection, the search for pulsating sdBs is quite time consuming, because only about $1 \%$ of the sdBs are variable. Therefore we apply a spectroscopic selection beforehand, searching for sdBVs with parameters near the instability strip. This preselection is based on the work of Edelmann et al. (2002) and Heber et al. (1999) who determined effective temperatures and surface gravities of sdBs from the Hamburg Schmidt survey (Hagen et al. 1995). This strategy has already led to the discovery of eight pulsating sdB stars (Silvotti et al. 2000; Østensen et al. 2001a,b) and we now present a new member of the sdBV group, HS0702+3206 with a photographic magnitude derived from the HS plates of $B=14^{\mathrm{m}} 7$.

Accurate spectroscopic parameters are not only necessary for the candidate selection, but also for further investigations. In the following we first present the spectroscopic results as derived from the model atmosphere analysis of optical spectra (Sect. 2), then the photometric observations (Sect. 3), and the analysis of the light curve (Sect. 4) of HS0702+3206. In Sect. 5 we discuss the results.

\section{Spectroscopy}

HS0702+3206 was classified as an sdB star by means of a low resolution spectrum $(7 \AA)$ taken with the Focal Reducer at the Calar Alto $3.5 \mathrm{~m}$ telescope. M. Altmann (Universiätssternwarte Bonn) kindly provided a medium
Table 1. Details of spectroscopic observations at the Calar Alto $3.5 \mathrm{~m}$ and $2.2 \mathrm{~m}$ telescopes.

\begin{tabular}{lcrcc}
\hline \hline star & Date & $\begin{array}{r}T_{\exp } \\
{[\mathrm{s}]}\end{array}$ & Instrument & $\begin{array}{c}\text { dispersion } \\
{[\AA / \mathrm{mm}]}\end{array}$ \\
\hline HS0702+6043 & $09 / 10 / 90$ & 1200 & FR+grism & 136 \\
HS0702+6043 & $27 / 11 / 00$ & 1200 & CAFOS & 100 \\
\hline
\end{tabular}

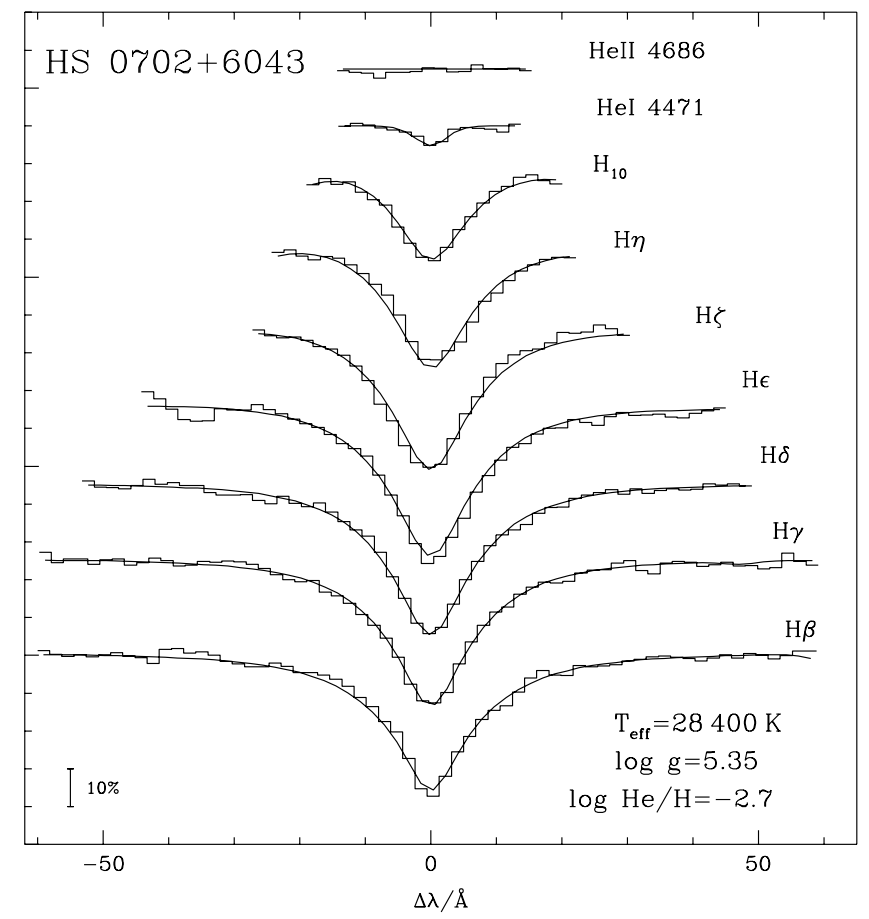

Fig. 1. Model fit to the Balmer and He line profiles in our time-averaged optical spectrum of HS0702+6043 using NLTE model atmospheres (see Sect. 2).

resolution spectrum obtained at the Calar Alto $2.2 \mathrm{~m}$ telescope equipped with the CAFOS spectrograph (see Table 1 for details of the observations). The improved spectral resolution $(5.5 \AA)$ as well as the larger spectral range of the latter spectrum enabled a more detailed spectral analysis. Besides the Balmer lines of hydrogen, He I lines are present whereas He II $4686 \AA$ is not found. No indication of a cool companion to this $\mathrm{sdB}$ star can be found in the spectrum. The exposure time is much longer than its oscillation periods (see Sect.4), therefore the spectrum is time-averaged.

A grid of synthetic spectra, derived from $\mathrm{H}-\mathrm{He}$ line blanketed NLTE model atmospheres (Napiwotzki 1997) was matched to the data (Fig. 1) to determine effective temperature, gravity and helium abundance simultaneously (see Heber et al. 2000a).

The formal fitting errors are small, i.e. $\Delta T_{\text {eff }}=$ $\pm 200 \mathrm{~K}, \Delta \log g= \pm 0.03$ and $\Delta \log (N(\mathrm{He}) / N(\mathrm{H}))=$ \pm 0.03 . Systematic errors, on the other hand, can only be quantified by repeated observations, but contribute significantly to the error budget. This led us to adopt the 


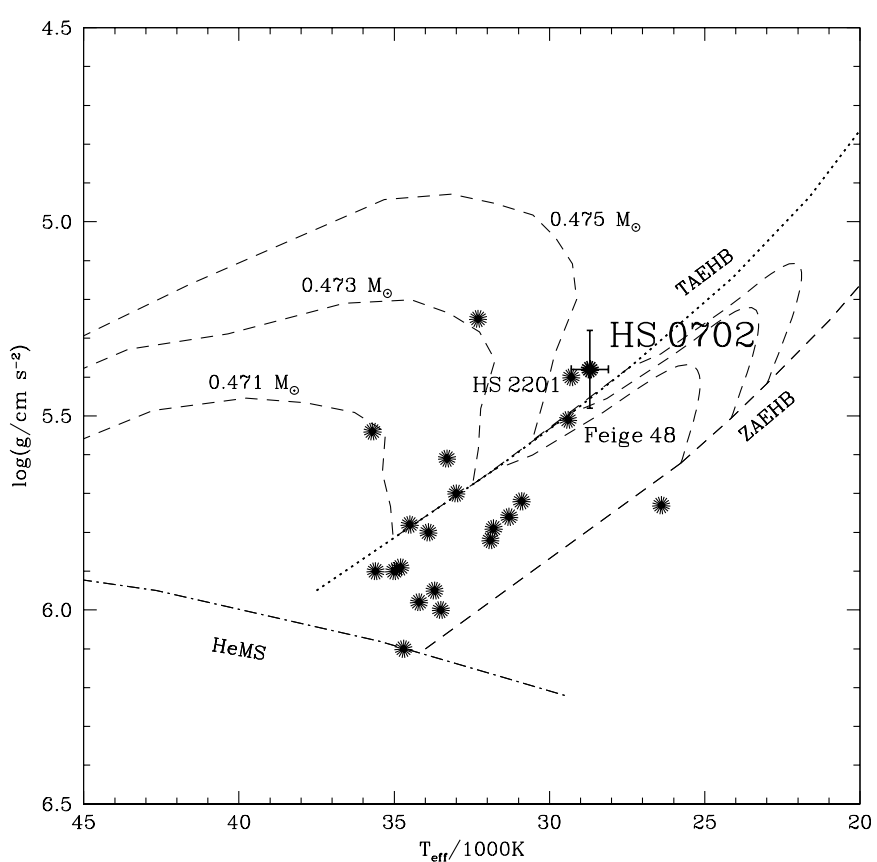

Fig. 2. Comparison of HS0702+6043 to previously known sdBVs in the $\left(T_{\text {eff }}, \log g\right)$ plane. Feige 48 and HS2201+2610 are marked for comparison. The position of the zero age extreme Horizontal Branch (ZAEHB), the terminal age extreme Horizontal Branch (TAEHB), the helium Main Sequence and evolutionary tracks for EHB stars (Dorman et al. 1995) are also shown.

following parameters: $T_{\text {eff }}=28400 \pm 600 \mathrm{~K}, \log g=$ $5.35 \pm 0.1, \log (N(\mathrm{He}) / N(\mathrm{H}))=-2.7 \pm 0.1$.

The parameters of HS0702+6043 place this star in the theoretical instability strip of pulsating sdB stars, as can be seen from a comparison to known pulsating sdB stars (see Fig. 2). The atmospheric parameters of HS0702+6043 are very similar to the parameters of the pulsating $\mathrm{sdB}$ stars Feige 48 and HS 2201+2610 (Heber et al. 2000b; Koen et al. 1998; Østensen et al. 2001a), which allows a comparison of the pulsation periods in relation to the spectroscopic parameters (see Sect. 5 ).

\section{Photometric time series}

\subsection{Observations}

In three observing runs we obtained time resolved CCD photometry for $14 \mathrm{sdBV}$ candidates. The data were obtained with the $1.23 \mathrm{~m}$ and $2.2 \mathrm{~m}$ telescopes at the DSAZ, Calar Alto, Spain, both equipped with blue-sensitive, back-illuminated SITe chips. The observations were made in white light at the $1.23 \mathrm{~m}$, i.e. without a filter, as good time resolution is crucial for the detection of the short periods, which implies short exposure times, and in white light or Johnson $B$ at the $2.2 \mathrm{~m}$. Likewise, the readout process was sped up by binning, either $2 \times 2$ or $3 \times 3$, resulting in 1 or 1.5 arcsec pixel sizes, respectively. The most significant reduction of the readout time could be achieved by making use of the windowing option, i.e. reading only one small section of the chip (containing the target, sky, and at least one comparison star).

In the first run in May 1999, we observed each target for about $1.5 \mathrm{~h}$ and if possible repeated the observation in one of the following nights. In the successive runs in December 1999 and November 2000, we extended the time span for each object in order to gain more confidence in the detection. In all three observing campaigns the weather conditions were poor.

\subsection{Data reduction}

Data reduction was carried out using our IDL software TRIPP (Time Resolved Imaging Photometry Package, see Schuh et al. 1999), which is based on the "CCD" photometry routines written by R. D. Geckeler (1998). TRIPP performs aperture photometry, which gives better results compared to point-spread-function fitting in un-crowded fields (O'Donoghue et al. 2000). The most important step is the calculation of the relative flux of a target with respect to one or more comparison stars. Only the simultaneous recording of comparison stars and sky background enables the detection of variations of the order of a few mmag at all; but this even under moderate conditions.

\section{Analysis of the time series}

The generally very low amplitudes often inhibit an immediate recognition of the presence of pulsations in a lightcurve. We apply different techniques to search for variability in the datasets: TRIPP allows the calculation of periodograms or an epoch folding approach, as well as fits with multiple sine functions.

Our data can be unevenly sampled, on a larger scale due to gaps from instrument or weather problems, and on smaller scales due to irregularities introduced by the operating system of the CCD control computer. Instead of rebinning the data we calculate a Lomb-Scargle periodogram according to Scargle (1982). This definition can take into account uneven sampling and provides a linear least-square fit to the data of the form $A \cos \omega t+B \sin \omega t$ for all frequencies $\omega$ of interest. We use the fast implementation following Press \& Rybicki (1989) in order to keep the computation time in moderate limits for large data sets. Using this fast algorithm also allows to calculate a statistical estimate of detection limits for every individual run. We simulate a large number of lightcurves with timing identical to the observation and assume white noise with the measured variance of the given observation. The highest peak of every individual periodograms is stored, then they are sorted monotonically. Comparing the height of a peak in the observation's periodogram with this accumulation of noise peaks therefore allows to derive confidence levels in the following sense: Whenever an observed peak at any arbitrary frequency point surpasses $90 \%$ of the noise peaks (collected over the whole frequency range), the probability for it being a false detection is less than $10 \%$. 


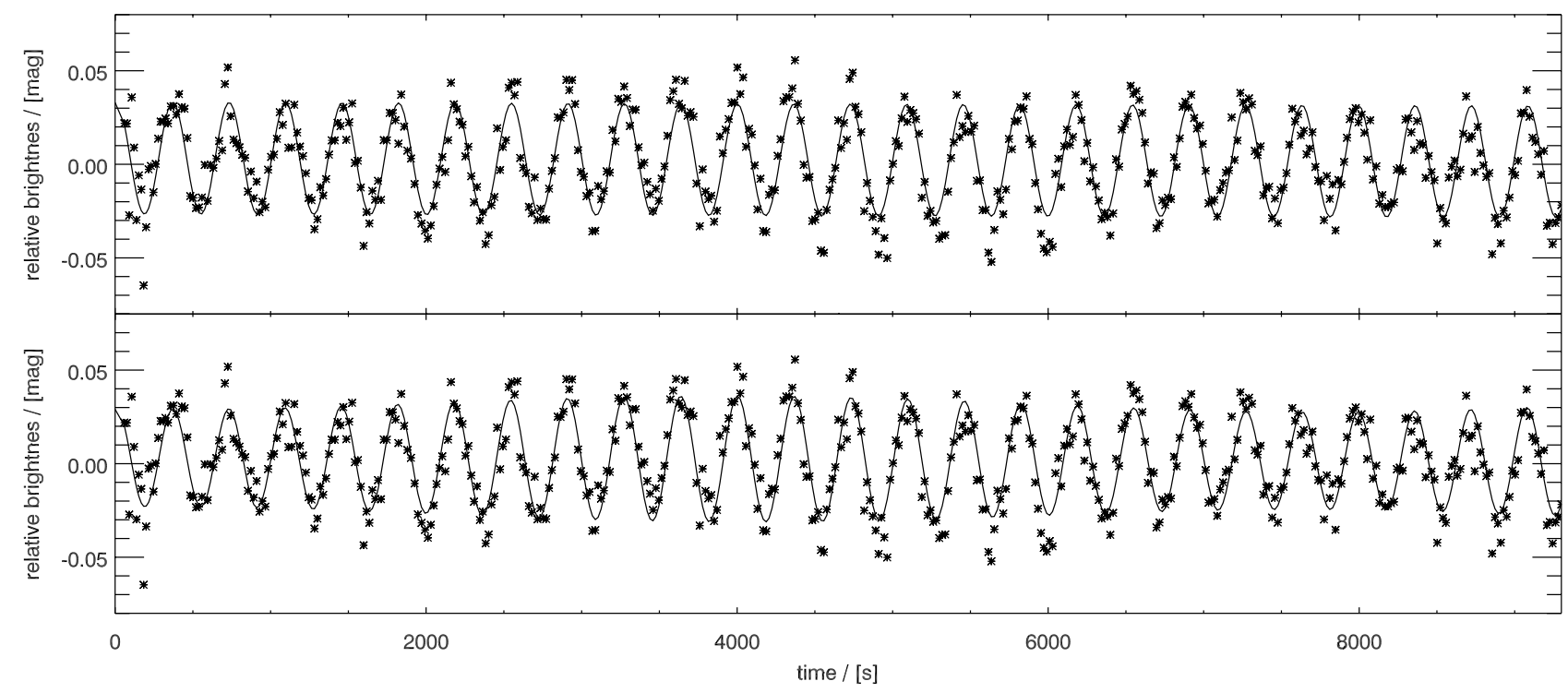

Fig. 3. Light curve of HS0702+6043: top: part of the light curve ("*") of HS0702+6043 overlayed with a sine fit ("-"; period = $363 \mathrm{~s}$, amplitude $=29 \mathrm{mmag})$. Time is measured in seconds since the start of the observation. Bottom: light curve and a sine fit with two periods (363/382 s, 29/3.8 mmag).

Table 2. Details of time series photometry at the Calar Alto $1.23 \mathrm{~m}$ telescope.

\begin{tabular}{lccrrr}
\hline \hline star & Date UT & $\begin{array}{r}T_{\text {exp }} \\
{[\mathrm{s}]}\end{array}$ & $\begin{array}{r}T_{\text {cycl }} \\
{[\mathrm{s}]}\end{array}$ & $\begin{array}{r}\text { Duration } \\
{[\mathrm{s}]}\end{array}$ \\
\hline HS0702+6043 & $07 / 12 / 99$ & $01: 17$ & 10 & 16.0 & 17855 \\
HS0702+6043 & $08 / 12 / 99$ & $04: 28$ & 8 & 15.5 & 6888 \\
HS0702+6043 & $09 / 12 / 99$ & $04: 45$ & 10 & 21.0 & 5020 \\
\hline
\end{tabular}

The epoch folding simply offers an independent check for any periodogram peak detection. In the case of a possible detection, we furthermore simulate a light curve with the same time sampling as the observation, with its observational noise characteristics and with all suspected periods at the derived amplitudes. We consider this to be the most direct method to assess the effects of the observation's window function.

We found one new sdBV, HS0702+6043, which will be further discussed in the following. Table 2 provides the observing log for the new sdBV star. The data set of HS0815+4243 confirmed the previously discovered variability of this star (Østensen et al. 2001a); the results from the remaining observations of eleven stars are further detailed at the end of this section (Table 3 ).

\section{1. $\mathrm{HSO} 0702+6043$}

The pulsation in HS0702+6043 became obvious already from its first light curve (Fig. 3). Therefore we observed the star on three consecutive nights to increase the time resolution.
The periodogram evidently shows a clear detection (Fig. 4). Both the sine fit and the period folding result in a period of $363 \mathrm{~s}$. The corresponding amplitude of $29 \mathrm{mmag}$ is among the largest of sdBVs. The periodogram of the pre-whitened light curve (Fig. 5) of HS0702+6043 reveals the presence of a second period at $382 \mathrm{~s}$ with an amplitude of $3.8 \mathrm{mmag}$. The power of the residual is significantly reduced if two periods are included in the simulation.

The presence of a second period is more difficult to obtain from the sine fit directly. Due to the large difference in the amplitude the fit does not look significantly better (Fig. 3), the reduced $\chi^{2}$, however, is slightly improved (1.01 instead of 1.03) when a second period is allowed for but does not further improve with more periods. As the separation of the two periods $(\Delta=19 \mathrm{~s})$ is considerably larger than the time resolution of the threedays run $(\Delta \approx 1.5 \mathrm{~s}$ at $363 \mathrm{~s})$, we regard both periods in HS0702+6043 as real.

\subsection{Detection limits for the other observations}

Periodogram analysis is well suited to pick out lowamplitude periods in noisy data: Horne \& Baliunas (1986) report that sinusoidal signals, if present, can still easily be detected if the signal-to-noise ratio is as low as 1:3. Even so, the noise level introduced to a lightcurve by anything from unfavorable weather to the contributions from photon statistics of course imposes an amplitude limit for a reliable exclusion of pulsations. The pulsation amplitudes of sdB stars are generally low (of the order of mmag) and could very well extend into unmeasurable ranges. We therefore deemed it reasonable to give the measured noise of all those observations where we did not detect any periodic intensity variations (Table 3 ). For comparison, we also determined the expected standard deviation for a 


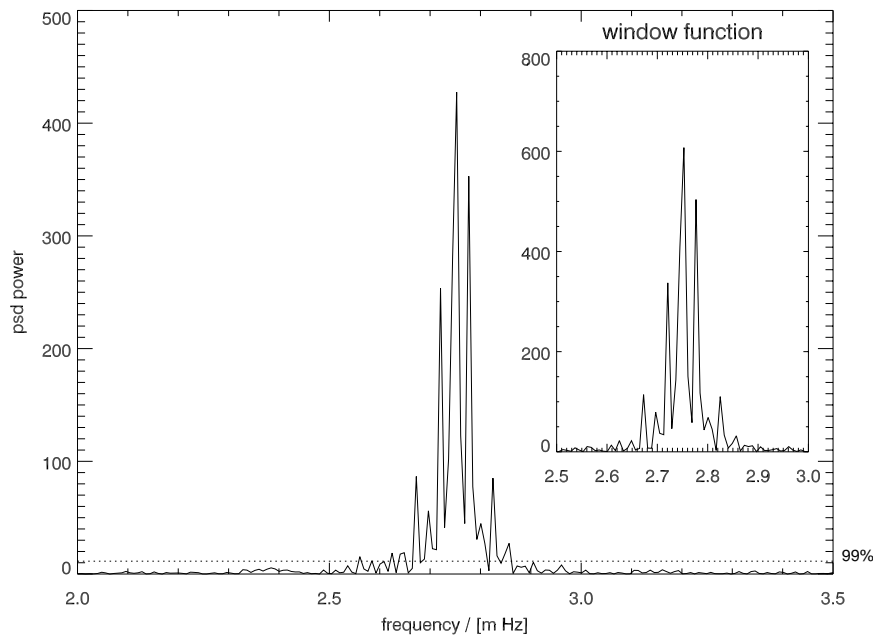

Fig. 4. Lomb-Scargle periodogram for HS0702+6043. The power spectral density (psd) is a measure for the probability that a period is present in the lightcurve. The horizontal line gives the confidence limit of $99 \%$.

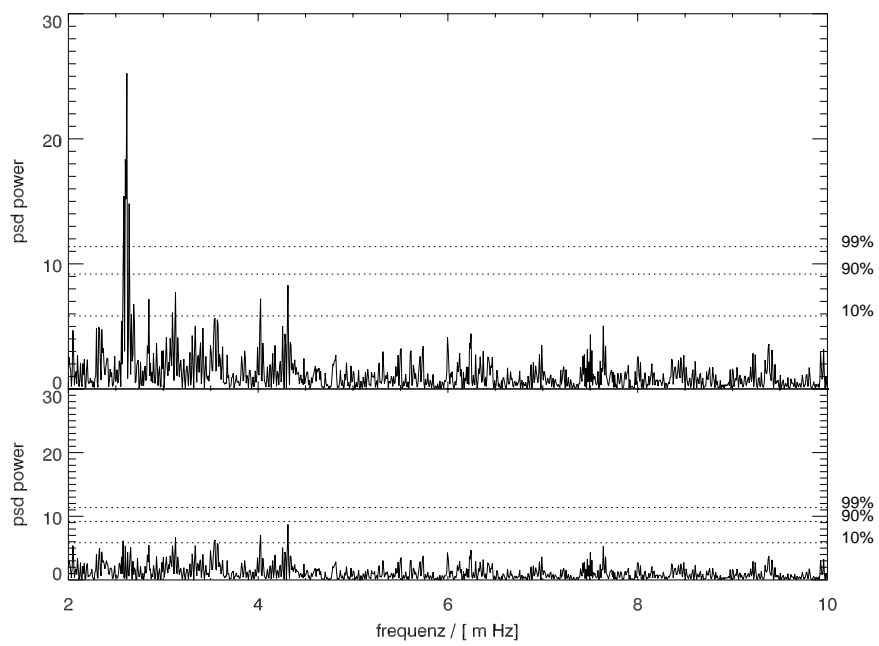

Fig. 5. Lomb-Scargle periodogram of the pre-whitened light curve of HS0702+6043: the sinusoidal fit with the dominant period is subtracted from the light curve in the upper panel whereas the two periods are subtracted from the light curve in the lower panel. The horizontal lines give the confidence limit of 10,90 , and $99 \%$.

stable star. For data sets with multiple comparison stars we interpolated the standard deviation - brightness relation to the brightness of the target star. The expected and measured standard deviations are consistent for most observations where we could determine an expectation value. HS0741+3818 and HS2100+1710, however, show a standard deviation which is a factor two higher than expected but together with our simulations of the confidence levels no indication for periodic variations could be found in those two stars. A re-observation might be useful in those cases. Any period with an amplitude as large as a third of the given standard deviation was definitely not present at the time of observation in the typical period range of minutes. Our observations put quite

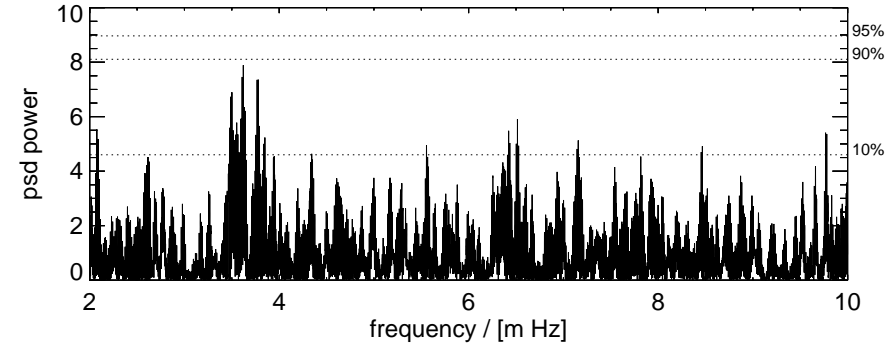

Fig. 6. Lomb-Scargle periodogram for HS0233+3037 of the December 5-8, 1999 data. The horizontal line gives the confidence limits of $95 \%, 90 \%$, and $10 \%$.

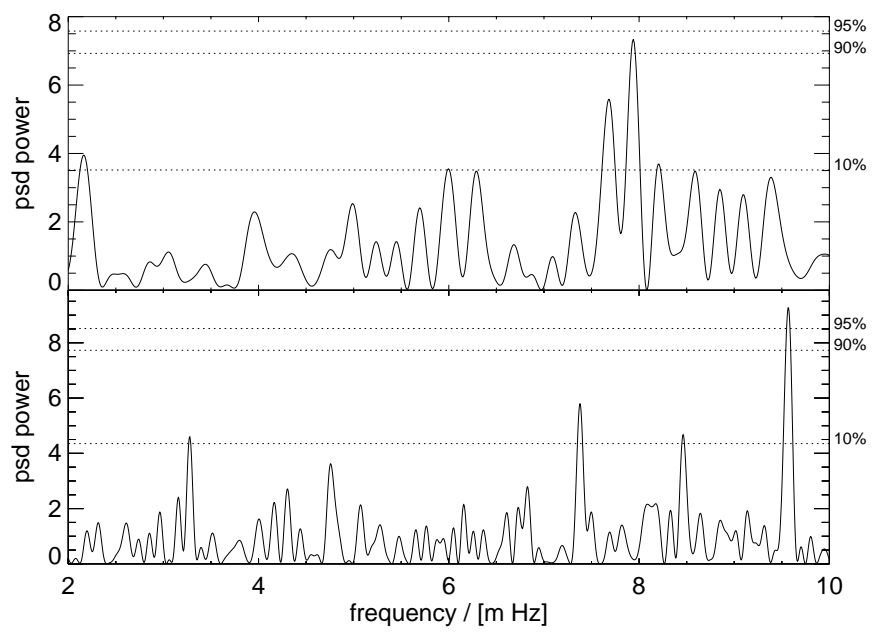

Fig. 7. Lomb-Scargle periodogram for HS2242+3206 of the May 1999 (top) and October 2000 (bottom) observation. The horizontal line gives the confidence limits of $95 \%, 90 \%$, and $10 \%$.

stringent limits on the pulsation amplitudes (few mmag) of HS0233+3037 (see Fig. 6), HS0741+3818, PG1649+356, HS1741+2133, and HS1831+6432, whereas the limit for HS1806+5024 is far too large to be meaningful. This star needs to be re-observed. In one object, HS2242+3206, we found possible evidence for low amplitude pulsation $(126 \mathrm{~s}$ amplitude $=1.7 \mathrm{mmag})$ with a confidence level near $2 \sigma$ (Fig. 7 ). Re-observation with the $2.2 \mathrm{~m}$ telescope, however, could not confirm this period but instead variation with a $2 \sigma$ significance (amplitude $=$ $1.3 \mathrm{mmag}$ ) was detected at a period of $105 \mathrm{~s}$. If these periods were real, this star would not fit in the period-density relation discussed below. Additionally, the expected and measured standard deviation agree. We therefore regard this star as stable unless further observations confirm one of the periods.

\section{Discussion}

A fundamental property of stellar pulsations is the perioddensity relation, because the hydrostatic time scale increases with decreasing density (e.g. Kippenhahn \& Weigert 1994). Such a relation could also be confirmed for the variable sdB stars (Østensen et al. 2001b), where the period of the largest pulsation mode is compared with the 
Table 3. Detection limits for the other observations. Only objects assumed to be non-variable are listed. Observation dates are given for the beginning of the night regardless of when exactly during the night an exposure series was started. " $B$ " is short for Johnson $B$, "-" stands for no filter. $N$ is the number of frames taken, $\sigma$ gives the standard deviation in units of mmag; $\sigma_{\exp }$ provides the expected standard deviation for a stable star.

\begin{tabular}{lcccrrr}
\hline \hline Object & Date & Tel. & Filter & $N$ & $\sigma$ & $\sigma_{\text {exp }}$ \\
\hline HS0233+3037 & $12 / 03 / 99$ & $1.2 \mathrm{~m}$ & - & 908 & 41 & $/$ \\
& $12 / 05 / 99$ & $1.2 \mathrm{~m}$ & - & 978 & 9 & 5 \\
& $12 / 06 / 99$ & $1.2 \mathrm{~m}$ & - & 688 & 6 & 6 \\
& $12 / 08 / 99$ & $1.2 \mathrm{~m}$ & - & 201 & 6 & $/$ \\
& $10 / 31 / 00$ & $2.2 \mathrm{~m}$ & $B$ & 338 & 8 & 4 \\
& $11 / 04 / 00$ & $2.2 \mathrm{~m}$ & - & 125 & $\approx 150$ & $/$ \\
HS0357+0133 & $12 / 11 / 99$ & $1.2 \mathrm{~m}$ & - & 496 & 19 & $/$ \\
& $11 / 01 / 00$ & $2.2 \mathrm{~m}$ & $B$ & 90 & 8 & 8 \\
HS0741+3818 & $12 / 10 / 99$ & $1.2 \mathrm{~m}$ & - & 957 & 12 & $/$ \\
& $12 / 11 / 99$ & $1.2 \mathrm{~m}$ & - & 300 & 12 & 6 \\
& $11 / 03 / 00$ & $2.2 \mathrm{~m}$ & $B$ & 281 & 9 & $/$ \\
PG1543+629 & $05 / 27 / 99$ & $1.2 \mathrm{~m}$ & - & 250 & 9 & 9 \\
PG1649+356 & $05 / 24 / 99$ & $1.2 \mathrm{~m}$ & - & 250 & 7 & 8 \\
HS1741+2133 & $05 / 21 / 99$ & $1.2 \mathrm{~m}$ & - & 250 & 6 & 6 \\
HS1806+5024 & $05 / 25 / 99$ & $1.2 \mathrm{~m}$ & - & 240 & $\approx 500 \approx 500$ \\
HS1831+6432 & $05 / 24 / 99$ & $1.2 \mathrm{~m}$ & - & 340 & 8 & 8 \\
HS2100+1710 & $05 / 21 / 99$ & $1.2 \mathrm{~m}$ & - & 250 & 12 & 7 \\
HS2144+225 & $05 / 20 / 99$ & $1.2 \mathrm{~m}$ & - & 200 & 7 & 7 \\
HS2202+2101 & $05 / 22 / 99$ & $1.2 \mathrm{~m}$ & - & 250 & 13 & 11 \\
HS2242+3206 & $05 / 22 / 99$ & $1.2 \mathrm{~m}$ & - & 230 & 5 & 5 \\
& $10 / 31 / 00$ & $2.2 \mathrm{~m}$ & - & 300 & 4 & $/$ \\
\hline \hline
\end{tabular}

density of the star as derived from spectroscopic parameters. Such a comparison implies that the largest modes are identical in all stars, i.e. belong to the same $l$ and $k$ numbers. The new sdBV HS0702+6043 is a spectroscopic twin to Feige 48 and HS2201+2610, i.e. it has an effective temperature and surface gravity similar to the latter two (see Fig. 2). The periods of HS0702+6043 are very similar to those of Feige $48(340-380$ s, Koen et al. 1998) and HS2201+2610 (350s, Østensen et al. 2001a). This star therefore fits well into that relation.

A comparison between observed and predicted periods provides the necessary tests for the predicted driving mechanism. HS0702+6043 is an important contribution since it is the coolest sdBV know today. Charpinet (2001) confronts his models with the most recent observations including HS0702+6043. The good agreement between theory and observation supports the current picture of the oscillations in sdBV stars, namely that the pulsations are driven by the $\kappa$-mechanism due to an opacity bump caused by iron resulting in radial and non-radial $p$-modes.
Since only two modes are observed in HS0702+6043, the potential of this star for detailed asteroseismology is low. However, the large amplitude in combination with a relatively long pulsation period make this star interesting for a detection of radial velocity variations caused by the pulsation which would then enable an alternative approach towards mode identification.

Acknowledgements. The authors would like to thank Thomas Rauch for supporting the observations in May, Jörn Wilms, Katja Pottschmidt and Sara Benlloch-Garcia for their support in the time series analysis, Ralf D. Geckeler for his photometry routines "CCD" and Martin Altmann for the spectrum of HS $0702+6043$. We would also like to thank the staff of the Calar Alto Observatory for the support during the observations. This research is supported by the DFG (HE 1354/301, DR 281/11-1, DR 281/12-1). S.D. and U.H. thank Dieter Engels, Hans-Jürgen Hagen and Dieter Reimers (Hamburg) for a fruitful collaboration over many years.

\section{References}

Billères, M., Fontaine, G., Brassard, P., et al. 2000, ApJ, 530, 441

Brassard, P., Fontaine, G., Billères, M., et al. 2001, ApJ, 563, 1013

Charpinet, S. 2001, AN, 322, 387

Charpinet, S., Fontaine, G., Brassard, P., \& Dorman, B. 1996, ApJ, 471, L103

Charpinet, S., Fontaine, G., \& Brassard, P. 2001, PASP, 113, 775

Dorman, B., Rood, R. T., \& O'Connell, R. W. 1993, ApJ, 419, 596

Dorman, B., O'Connell, R. W., \& Rood, R. T. 1995, ApJ, 442, 105

Edelmann, H., Heber, U., Napiwotzki, R., et al. 2002, A\&A, in prep.

Geckeler, R. D. 1998, Ph.D. Thesis, Eberhard-KarlsUniversität Tübingen

Hagen, H. J., Groote, D., Engels, D., \& Reimers, D. 1995, A\&AS, 111, 195

Heber, U. 1986, A\&A, 155, 33

Heber, U., Edelmann, H., Lemke, M., Napiwotzki, R., \& Engels, D. 1999a, in 11th European Workshop on White Dwarfs, ed. J.-E. Solheim, \& E. Meištas, ASP Conf. Ser., 169,551

Heber, U., Reid, I. N., \& Werner, K. 2000a, A\&A, 348, L25

Heber, U., Reid, I. N., \& Werner, K. 2000b, Baltic Astron., 9, 171

Heber, U., Moehler, S., Napiwotzki, R., \& Thejll, P. 2002, $\mathrm{A} \& \mathrm{~A}$, in press [astro-ph/0201096]

Horne, J. H., \& Baliunas, S. L. 1986, ApJ, 302, 757

Kawaler, S. D., \& Bradley, P. A. 1994, ApJ, 427, 415

Kilkenny, D., Koen, C., O'Donoghue, D., \& Stobie, R. S. 1997, MNRAS, 285, 640

Kippenhahn, R., Weigert, A. 1994, Stellar Structure and Evolution, 3rd edition (Springer, Berlin)

Koen, C., O'Donoghue, D., Pollacco, D. L., \& Nitta, A. 1998, MNRAS, 300, 1105

Napiwotzki, R. 1997, A\&A, 322, 256

Maxted, P. F. L., Heber, U., Marsh, T. R., \& North, R. C. 2001, MNRAS, 326, 1391 
O'Donoghue, D., Koen, C., Kilkenny, D., Stobie, R. S., \& Lynas-Gray, A. E. 1999, in 11th European Workshop on White Dwarfs, ed. J.-E. Solheim, \& E. Meištas, ASP Conf. Ser., 169, 149

O'Donoghue, D., Kanaan, A., Kleinman, S. J., Krzesinski, J., \& Pritchet, C. 2000, Baltic Astron., 9, 375

Østensen, R., Solheim, J. E., Heber, U., et al. 2001a, A\&A, 368, 175

Østensen, R., Heber, U., Silvotti, R., et al. 2001b, A\&A, 378, 446

Piccioni, A., Bartolini, C., Bernabel, S., et al. 2000, A\&A, 354, L13

Press, W. H., \& Rybicki, G. B. 1989, ApJ, 338, 277
Saffer, R. A., Green, E. M., \& Bowers, T. P. 2001, in 12th European Workshop on White Dwarfs, ed. J. L. Provencal, H. L. Shipman, J. MacDonald, \& S. Goodchild, ASP Conf. Ser., 226, 408

Scargle, J. D. 1982, ApJ, 263, 835

Schuh, S. L., Dreizler, S., Deetjen, J. L., Heber, U., \& Geckeler, R. D. 1999, Baltic Astron., 9, 395

Silvotti, R., Solheim, J. E., Gonzalez Perez, J. M., et al. 2000, A\&A, 359, 1068

Ulla, A., Zapatero Osorio, M. R., Pérez Hernández, F., MacDonald, J. 2001, A\&A, 369, 986

Yi, S., Demarque, P., \& Oemler, A. J. 1997, ApJ, 486, 201 